\title{
Size effects in spin-crossover nanoparticles in framework of 2D and 3D Ising-like breathing crystal field model
}

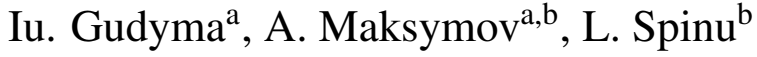 \\ ${ }^{a}$ Department of General Physics, Chernivtsi National University, Chernivtsi 58012, Ukraine \\ ${ }^{b}$ Advanced Material Research Institute (AMRI) and Department of Physics, University of New Orleans, New Orleans, LA 70148, USA
}

\begin{abstract}
The spin-crossover nanoparticles of different sizes and stochastic perturbations in external field taking into account the influence of the dimensionality of the lattice was studied. The analytical tools used for the investigation of spin-crossover system are based on an Ising-like model described using of the breathing crystal field concept. The changes of transition temperatures characterizing the systems' bistable properties for 2D and 3D lattices, and their dependence on its size and fluctuations strength were obtained. The state diagrams with hysteretic and non-hysteretic behavior regions have also been determined.
\end{abstract}

(C) 2014 Published by Elsevier Ltd.

Keywords: spin-crossover, Ising model, breathing crystal PACS: 05.40.-a, 75.30.Wx, 02.50.Ey

\section{Introduction}

In the last decades the attractiveness of magnetic molecular nanoparticles has been increased and their theoretical and experimental studies have been intensified [1-5]. Among the prospective materials which are able to satisfy the requirements of contemporaneous nanoelectronics are spin-crossover compounds. This is the class of magnetic molecular complexes based on transition metal ion with electronic configuration varying between $d^{4}-d^{7}$ and surrounded by nonmetallic ligand environment $[6,7]$. The main focus of this research is on iron(II) based spin-crossover systems, which are characterized by octahedral symmetry of ligand field and the splitting of $d$-orbitals into sublevels with two irreducible representations $t_{2 g}$ and $e_{g}$ with different spin configuration. The repartition of $d$-electrons on sublevels takes place according Hund's rule and depends on the ratio between ligand's field splitting energy and spin pairing energy. If this ratio is greater than 1 , i.e. the spin-pairing energy is small, the $t_{2 g}$ irreducible representation is energetically favorable where the electrons are paired with antiparallel spins which gives the minimal magnetic moment of the compounds and called low-spin (LS) state. If the ratio between ligands field splitting energy and spin pairing energy is less or equal to 1 the electrons pairing becomes unfavorable and the repartition of electrons over all sublevels takes place. In this case the high-spin (HS) configuration is realized with maximal magnetic moment. The schematic representation of electrons' distribution for particular spin configurations of spin-crossover molecule, which may be controlled by external stimuli is shown in Fig. 1. The macroscopic magnetic properties of these configurations are diamagnetic for LS state and paramagnetic for HS state. During transition, the magnetic moment change is also accompanied by a change in metal-ligand distance as shown in Fig. 1. One must note that the properties which vary during the spin-crossover phenomenon are not limited only to those mentioned above. The LS and HS spin 
configurations are also characterized by a range of properties which significantly differ for each state as molecular volume, color, magnetic susceptibility etc.
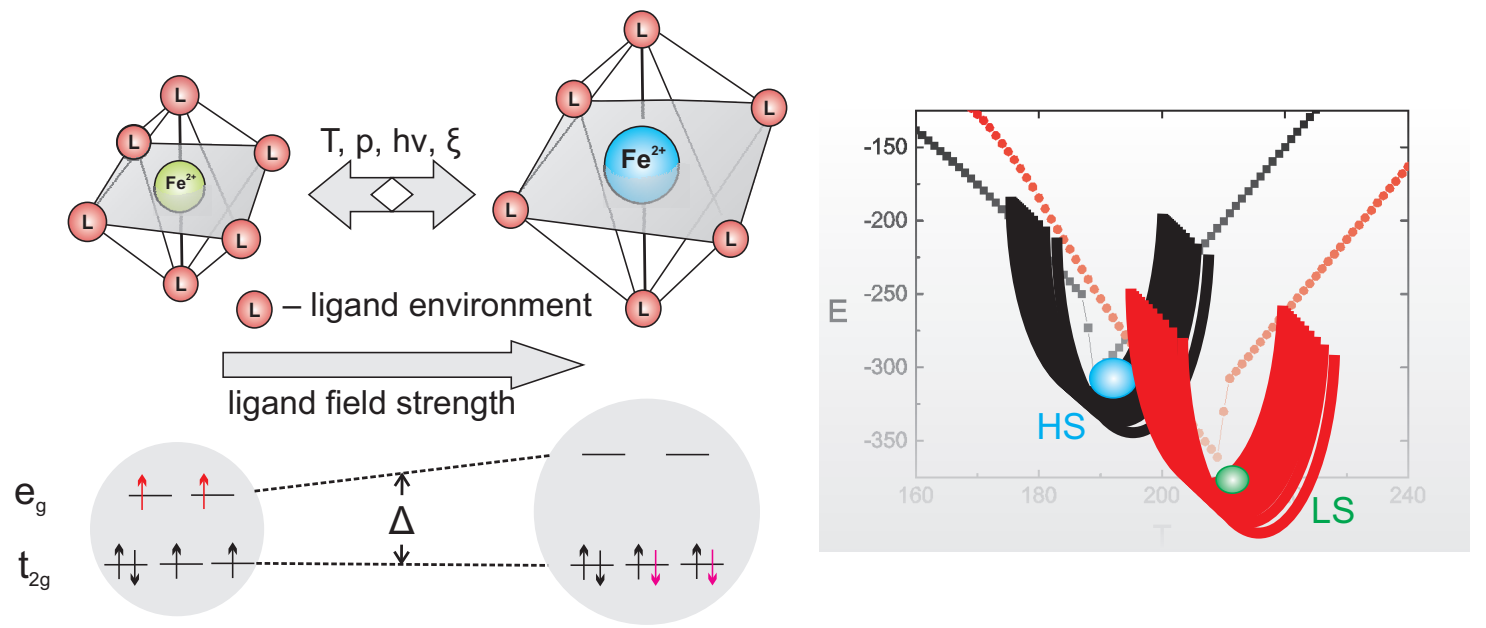

Figure 1. The variation of metal-ligand bound length under the influence of external stimuli. The properties of the material are determined by the specific distribution of the electrons on the d-orbitals sublevels.

The existence of two stable configurations leads to the commuting properties of spin-crossover materials. The bistability of spin-crossover compounds becomes indeed valuable with the possibility to change spin configuration by the action of external stimuli like temperature, light irradiation, magnetic field, pressure [8-13]. The possibility of controlling the transition between the spin states together with the hysteretic behavior give the possibility to use them in a wide range of electronic devices as sensors, data storage and recording systems, ultrafast switching devices $[6,7,14-19]$. In order to understand their functioning is very important to evaluate the behavior of molecular spincrossover nanoparticles in hysteretic and non-hysteretic regimes and to find out the conditions in which these regimes occur, especially in systems of finite size.

In this work we study a magnetic spin-crossover nanosystem in the framework of microscopic Ising-like model with finite size, taking into account the energy perturbations of the external field, relative to transition metal ion. The main goal is to find out the influence of finite size effects and fluctuations strength of ligand field on the hysteretic properties of the spin-crossover system. Thus, the transition temperatures of finite size spin-crossover nanoparticles are characterized and the state diagram with the regions of phase space with hysteretic and non-hysteretic behavior have been obtained.

\section{Ising-like spin-crossover model with breathing crystal field}

The behavior of spin-crossover compounds at molecular level may be described by the microscopic Ising-like model in the framework of mean field approach with the intermolecular interaction. The Hamiltonian of such model is the following $[20,21]$ :

$$
H=-J \sum_{\langle i j\rangle} s_{i} s_{j}-h_{0} \sum_{i} s_{i}
$$

Here, $s_{i, j}$ is a pseudospin scalar variable taking the values \pm 1 for LS and HS states, respectively, $J$ is the intersites coupling and $h_{0}$ is the external field relative to metal ion. For simplicity the intermolecular interaction in spincrossover materials is assumed to be the same for all sites in the system and in a phenomenological approach this is described as a ferromagnetic coupling $(J>0)$ between nearest neighboring spins. This is the simplest way to express the cooperativity between magnetic molecules of spin-crossover material [22-25].

The influence of external physical field on transition metal ion is described by the second term of Eq. (1). In this way it is possible to characterize the occurrence of intramolecular processes and describe the behavior of the 
spin-crossover compound under environmental influences. If we are taking into account only thermal activation of spin transition the external field $h_{0}$ may be written as:

$$
h_{0}=-(\Delta-k T \ln g),
$$

where $\Delta$ is the ligand field splitting energy, $k$ is Boltzmann constant, $T$ is temperature, and $g$ is the degeneracy ratio between HS and LS states.

According to point charge model approximation of the ligand field the energy gap between LS and HS states may be described in the following way

$$
\Delta=\frac{5}{3} \frac{Z e^{2}}{R^{3}}\left\langle r^{4}\right\rangle,
$$

where $Z e$ is the effective charge of the ligand, $R$ is the distance between metal ion and ligand environment, and $\left\langle r^{4}\right\rangle$ is the mean fourth-power radius of the $d$ electron. One has to notice that this relation is valid only within an electrostatic model and for perfect octahedral symmetry. In this case the external field $h_{0}$ is the same for all molecules in the sample and for all times. This approach works well for large highly-symmetric systems, but local and instantaneous thermal fluctuations break their symmetry, creating deviations from zero-order approximation, which can be treated by using statistical fluctuations. The symmetry breaking by influence of fluctuations is especially important for nanosystems with small particle size containing from one up to a few molecules. The concept of breathing crystal field description by statistical instantaneous fluctuations is based on the fact that the dynamics of thermal switching, which take place for an ensemble of spin-crossover sites, span the microsecond time scale, while the expansion of particular molecule occurs on nanosecond or even picoseconds time scale [26, 27].

The equations (2) and (3) offer a good characterization of the spin-crossover materials behavior in a zero-order approximation of the mean-field theory. Therefore, the model (1) must be improved for the case with "breathing" crystal field. This means that the external site-dependent field $h_{i}$ varies in time according to the relation $-h_{i}=$ $-h_{0}+\xi_{i}(t)$, which leads to the Hamiltonian of the next form [24]

$$
H=-J \sum_{\langle i j\rangle} s_{i} s_{j}-\sum_{i}\left[\Delta-k T \ln g+\xi_{i}(t)\right] s_{i} .
$$

From the point of view of Ising model, the stochastic process $\xi_{i}(t)$ reflects the external local random field statistically perturbed. This fact leads to the system's behavior during cooling and heating processes, that may differ significantly from the one for fluctuationless systems [24]. From Eq. (4), it may be observed that the role of the stochastic term becomes relevant, especially for critical temperatures.

In the simplest case, the fluctuations are uncorrelated and can be represented by a white Gaussian stochastic process. In this case, each site is considered that fluctuates separately and is not affected by the fluctuations of all the other sites of the system. The statistical characteristics of the fluctuations of a separate spin-crossover molecule can be written as $[28,29]$

$$
\langle\xi(t)\rangle=0,\left\langle\xi(t) \xi\left(t^{\prime}\right)\right\rangle=2 \varepsilon^{2} \delta\left(t-t^{\prime}\right) .
$$

Here, $\varepsilon$ is the strength of fluctuations and characterizes the spectral density of stochastic variable $\xi_{i}(t)$, while $t$ and $t^{\prime}$ are distinct times.

\section{Computational details of Monte Carlo simulations}

We have studied the behavior of a spin-crossover system with breathing crystal filed described by Hamiltonian (4) with conditions (5) and considering the influence of the ratio between the area of spin-crossover nanoparticle and its volume. The small particle is reasonable to be modeled with "open boundary conditions" which means that the interaction with sites from surface is zero. In this paper we will focus on the two-dimensional and three-dimensional spin-crossover models with square and cubic geometries, respectively and open boundary conditions. In the case of a three dimensional system, for the first term of hamiltonian (4), one needs to take into account the contribution of a large enough number of nearest neighbors for the mean field approximation. The analytical solution for three dimensional Ising-like systems is difficult to obtain and in the presence of stochastic processes only numerical simulations can be used for their characterization. 
The examination of spin-crossover system described by Hamiltonian (4) has been carried out by Monte Carlo simulations using standard Metropolis algorithm. The systems properties are characterized by the changes of its transition temperatures during cooling and heating processes. Besides the influence of size effects on transition temperatures, we have investigated their dependence on the strength of fluctuations that for small size open boundary systems becomes very relevant.

In order to find the transition temperature other system and environmental parameters were fixed. Thus, the temperature transition curves were obtained using the following Metropolis algorithm: (i) fix the temperature; (ii) fix initial spin configuration; (iii) find the system energy for initial configuration; (iv) flip arbitrarily one spin from the system; (v) find the system energy of new configuration; (vi) evaluate the transition probability of new configuration and determine the possibility of transition; (vii) if the transition is accepted the system magnetization is found, otherwise the spin configuration remains unchanged and the next Monte Carlo (MC) step follows, i.e. the algorithm is repeated from step (i). The spin-transition probability of arbitrarily chosen spin $s_{i}$ on step (vi) is described by the following expression:

$$
P\left(s_{i} \rightarrow-s_{i}\right)=\min \left[1, \exp \left(-\frac{\Delta H\left\{s_{i}\right\}}{k T}\right)\right],
$$

where $\Delta H\left\{s_{i}\right\}$ is the energy difference when a spin changes from $s_{i}$ to $-s_{i}$ :

$$
\Delta H\left\{s_{i}\right\}=2 J s_{i} \sum_{j} s_{j}-2 h_{i} s_{i} .
$$

For simulations $k=1$ was used for the Boltzmann constant.

The calculations of transition curves start from the high temperature state where all spins are "up", i.e. in HS state. One thermal cycle consists in a temperature sweep from the HS state to the LS, where all spins are "down", and back to initial HS state. The system's magnetization at each temperature is calculated as the average value of the magnetization over MC trajectory from stationary regime. The correspondence between the magnetization of Ising model and the order parameter of spin-crossover system, that is HS fraction $n_{H S}$, is given by the relation below:

$$
n_{H S}=\frac{m+1}{2}
$$

The transition temperature is defined as the temperature for which HS fraction $n_{H S}=0.5$ during cooling and heating of spin-crossover system.

\section{Results and Discussion}

In this section are shown the results of microscopic Monte Carlo modeling of the spin-crossover system as described above. We focus on the iron(II) based spin-crossover systems with six electrons on $d$-orbitals and ligand field splitting energy $\Delta=1000$, and degeneracy ratio $g=150$. These parameters correspond to an entire class of spincrossover complexes based on $F e^{2+}$ ion. The calculations were performed considering systems with a relatively small lattice size. Specifically, for the $2 \mathrm{D}$ systems, the lattice parameter changes from $L=3$ to 25 , while for $3 \mathrm{D}$ systems a lattice parameters from $L=3$ to $L=15$ were considered. In both cases 1000 Monte Carlo steps were used.

For characterizing the behavior of spin-crossover systems subject to fluctuations, we have calculated the dependence of transition temperatures $T_{d o w n}$ and $T_{u p}$ on the fluctuations strength and on the ratio between the area of spin-crossover nanoparticle and its volume. For a 2D lattice system the area $S$ of the spin-crossover nanoparticles is defined as the number of sites inside the lattice with the perimeter $P$. The results obtained for a $2 \mathrm{D}$ lattice with an intersites interaction $J=100$ are given in Fig. 2. In Fig. 2 the bottom surface corresponds to the cooling transition temperature $T_{\text {down }}$ and the top surface describes the behavior of heating transition temperature $T_{u p}$. The difference between $T_{u p}$ and $T_{\text {down }}$ gives information about hysteresis width. As it can be observed in Fig. 2 the width of thermal hysteresis, increases as both, fluctuation strength and ratio $P / S$, increase. This kind of behaviour is in agreement with previously reported results in the case of space-time correlated fluctuations [24]. However, the hysteresis expansion with increasing of fluctuations strength, as shown in Fig. 2, is strongly dependent on the lattice size. As was shown [24] there is a critical system size beyond which any further increase of its size does not affect the hysteresis width 

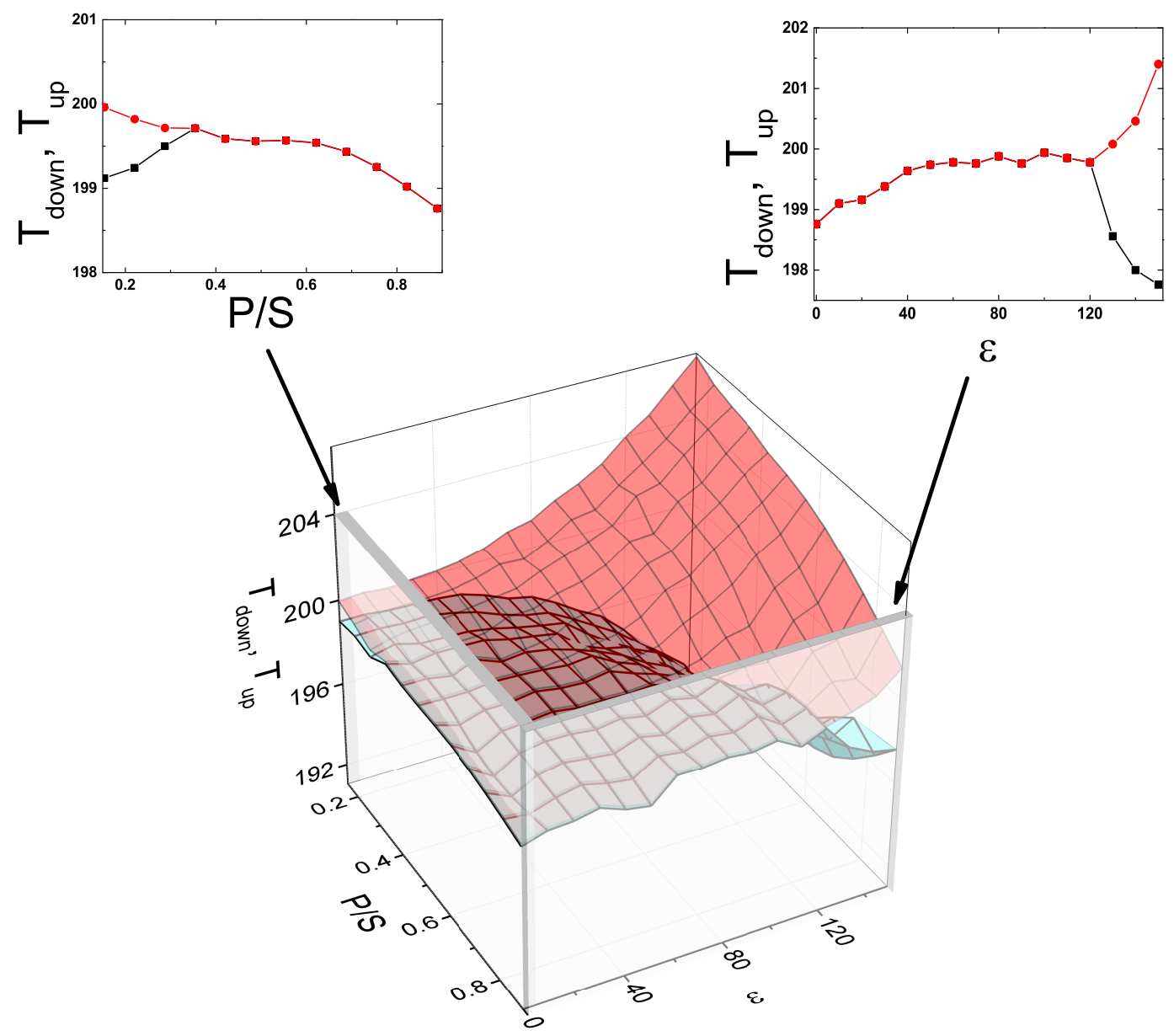

Figure 2. The transition temperatures during cooling and heating processes $T_{\text {down }}$ (online cyan surface) and $T_{u p}$ (online red surface), respectively of a 2D spin-crossover system with intersite interaction $J=100$. Each temperature value is obtained as an average over 25 hysteresis cycles.

of the system with open and periodic boundary conditions. For Ising-like fluctuationless model the value of hysteresis width is the result of intersites interactions only, which is the case for large enough (larger than its critical size) systems.

In order to better understand the behavior of transition temperatures for small lattices we chose a value for the intersites interaction $J$ in such a way that it determine a hysteretic behavior for fluctuationless system with a critical system size. We found that the difference between $T_{u p}$ and $T_{\text {down }}$ for fluctuationless system $(\varepsilon=0)$ vanishes for $P / S=0.33$ which corresponds to the lattice with $L=11$. The vanishing of hysteresis loop for fluctuationless case is additionally presented in the right top panel of Fig. 2. However, for the chosen intersite interaction $(J=100)$ one expects to have a nonzero hysteresis width. Such non-hysteretic behavior is caused mainly by the finite size of the system, especially by its open boundary. We theorize that the reduction of sites in the system leads to an almost instantaneous responses on external actions during cooling and heating, vanishing the hysteresis width. Besides this, for spin-crossover systems with small lattice the cooperative effects which are one of the main reasons of their hysteretic behavior is also decreased. In the investigated systems with small lattice size one needs to emphasize the role of fluctuations that tend to increase the hysteresis even when the number of molecules in the system is small. For the smallest lattice size which it was taken into account, i.e. $L=3$, the appearance of hysteresis is observed for $\varepsilon=120$ as is clearly seen on the top left panel of Fig. 2 . 
By decreasing the contribution of sites from open boundaries in interaction term, and therefore in thermal transition curves the hysteresis width is enlarged, as it is observed in Fig. 2 for small values of the ratio $P / S$. One observes that the hysteresis width is maximal for smallest ratio $P / S$ (i.e. for largest $L$ ) and for maximal fluctuation strength chosen in our simulations, i.e. $\varepsilon=150$.

In order to study the role of system dimensionality we have analyzed the transition temperatures for threedimensional Ising-like spin-crossover model with open boundary conditions, with increasing fluctuations strength $\varepsilon$, and considering the influence of size effects. Similar to the 2D system the finite size effects were characterized by the ratio between the number of sites on the system boundaries (area of cubic lattice $S$ ) and total number of sites (volume $V$ ). The results obtained for the same intersites interaction as for 2D system are presented in Fig. 3.

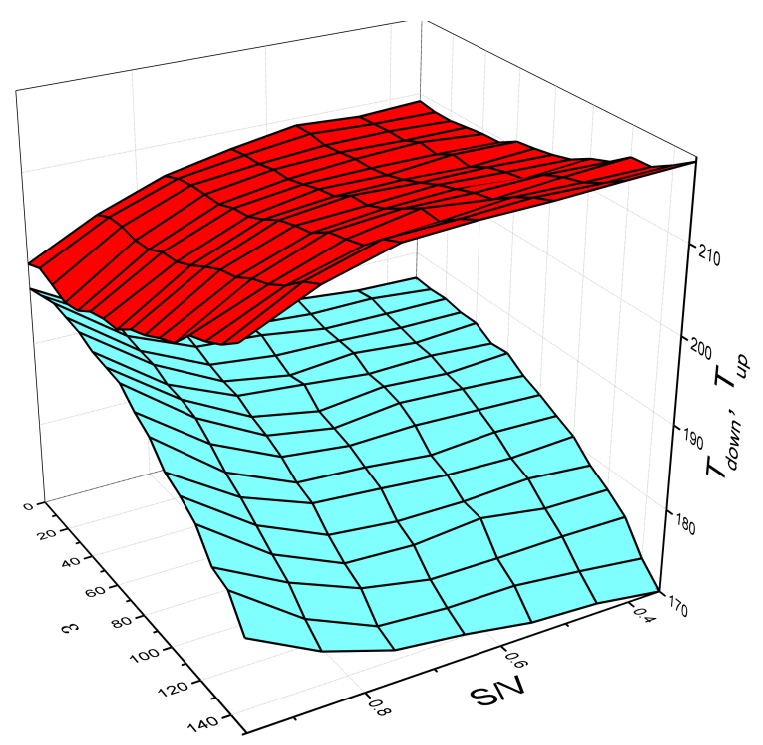

(a)

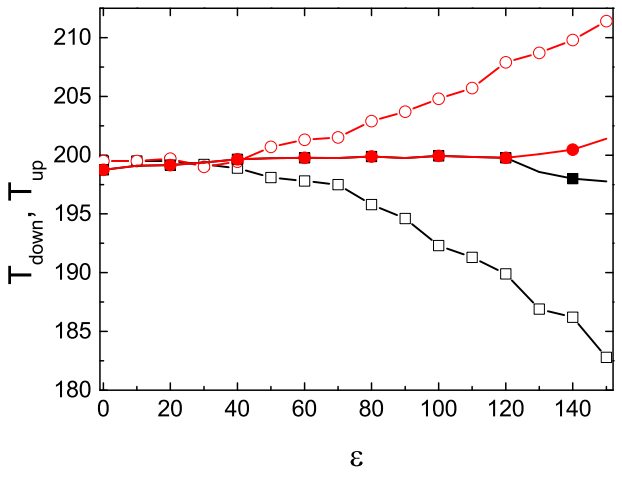

(b)

Figure 3. The transition temperatures during cooling $T_{\text {down }}$ (online cyan surface), and heating $T_{u p}$ (online red surface) processes for different system ratio $S / V$ and fluctuations strengths for the 3D spin-crossover model with the intersites interaction $J=100$ (a). The variation of transition temperatures as a function of $\varepsilon$ for the $2 \mathrm{D}$ and $3 \mathrm{D}$ systems is shown in (b). Each temperature value is obtained as an average obtained from 10 hysteresis cycles.

In Fig. 3 the values of transition temperatures during cooling form the bottom surface, whereas the ones during heating form the upper one. As we can see the behavior of hysteresis width for three-dimensional system with open boundary condition differs from the one for two-dimensional system. One of the main differences is the fact that for the fluctuationless case the non-hysteretic behaviour occurs for much larger ratio $S / V=0.96$ that corresponds to the smaller lattice with $L=3$. In contrast to the two-dimensional system with the same lattice value the presence of even slight fluctuations leads to a bistable behavior that may be observed from the strength $\varepsilon=30$. In the case of $3 \mathrm{D}$ systems a larger hysteresis width is obtained with increasing fluctuations strength. The comparison of the transition temperatures curves between 3D and 2D systems (open and full symbols, respectively) with lattice size $L=3$ is displayed in Fig. 3 (b). By analyzing the influence of fluctuations on the system with other lattice sizes it may be observed that in all cases the hysteresis width monotonically increases with increasing of fluctuation strength. The difference in system size and its dimensionality gives only quantitative changes of this increasing.

If we compare Figs. 2 and 3 we can see that the finite size effects for three-dimensional lattice are less pronounced than for $2 \mathrm{D}$ system, even if $S / V$, i.e. the contribution of the sites from the edges, is larger. For the 3D system it is observed a clear saturation tendency of the transition temperatures variation with decreasing $S / V$, whereas for the 2D lattice of same side sizes, a monotonic increasing of hysteresis width is obtained.

Due to the different behavior of the 2D and 3D systems mentioned above, the conditions for which the nonhysteretic behaviour occur in both cases is of great importance. The ability to distinct the regions with hysteretic and nonhysteretic behaviors makes possible to characterize the first-order and second order phase transition taking into account the main system parameters that define spin-crossover system state. The final state diagrams with the 
boundary between monostable non-hysteretic region and bistable hysteretic one are given in Fig. 4 (a) and (b) for 2D and 3D spin-crossover system lattice, respectively. The states diagrams in both cases were obtained by analyzing the hysteresis loops for which the values of lattice size $L$, fluctuations strength $\varepsilon$ and intersites interaction $J$ lead to disappearance of hysteretic behavior. Each point from the set that form the surface in Fig. 4 (a) were averaged over 15 executions for system lattice $3 \leq L \leq 5,10$ executions for $5<L \leq 9$ and 5 executions for $9<L \leq 25$. The similar averaging was provided for the curves from Fig. 4 (b) where each point was averaged over 10 executions for $L=3,5$ executions for $L=5$, and $L=7$. For any point chosen below the surface from Fig. 4 (a) and below the specific curve from Fig. 4 (b) the spin-crossover system shows non-hysteretic transitions during cooling and heating processes.

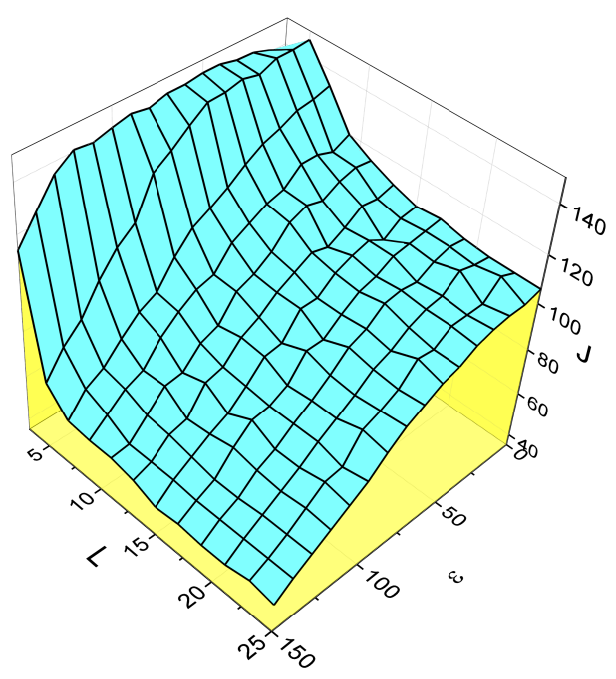

(a)

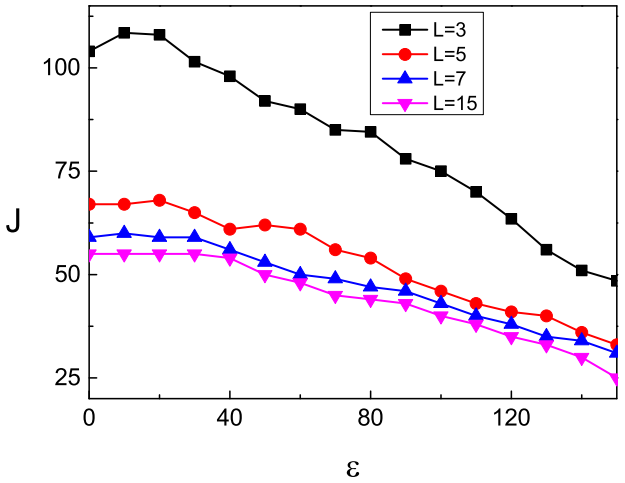

(b)

Figure 4. State diagram of spin-crossover system with 2D square lattice and 3D cubic lattice in the case of open boundary conditions.

For the studied spin-crossover model it may be observed that in the presence of small fluctuations, the occurrence of hysteresis is controlled only by intersites interaction, as shown by the insignificant dependence of $J$ on low fluctuations for each system size (see Fig. 4). From falling character of the plots shown in Fig. 4 it is observed that the role of fluctuations in appearance of hysteresis increases with increasing their strength, i.e. with increasing the fluctuations one needs smaller intersite interactions that lead to bistability. By comparing the Fig. 4 (a) and Fig. 4 (b) one observes that for the 2D system the dependence on size effects is more pronounced than for the 3D system, where the tendency to system saturation is observed when its size is increasing.

\section{Conclusion}

In this work we have provided the study of two-dimensional and three-dimensional spin-crossover magnetic nanoparticles, described by an Ising-like model in framework of breathing crystal field concept. The conditions of monostable and bistable behaviors of spin-crossover systems have been characterized and corresponding state diagrams with indication of the region of first-order and second order phase transitions have been built by using Monte Carlo simulations carried out in framework of Metropolis algorithm. Also, the detailed description of the dependencies of hysteresis width on the size effects and the strength of statistical fluctuations were provided. The contributions of the atoms from lattices edges of the 2D and 3D systems in their behavior were taken into account.

It is find out that increasing the fluctuation strength and lattice size side lead to an increase of the hysteresis width, which it is more pronounced for the three-dimensional system. In the case of cubic lattice the system dependence on its size achieves the saturation for smaller system size comparatively to the 2D system, where saturation on system size is not achieved for the chosen range of lattice side values. The decreasing of intersite interactions by increasing fluctuations strength, regardless of system dimensionality, emphasizes the role of fluctuations in the hysteretic behavior and it makes possible to obtain a spin-crossover system with bistable properties with weaker coupling between its sites. 


\section{Acknowledgments}

The work at University of New Orleans was supported by the National Science Foundation under the NSF EPSCoR Cooperative Agreement No. EPS-1003897 with additional support from the Louisiana Board of Regents.

\section{References}

[1] O. Kahn, C. J. Martinez, Spin-transition polymers: From molecular materials toward memory devices, SCIENCE 279 (1998) $44-48$.

[2] B. Weber, J. Obel, D. Henner-Vásquez, W. Bauer, Two new iron(II) spin-crossover complexes with $\mathrm{N}_{4} \mathrm{O}_{2}$ coordination sphere and spin transition around room temperature, Eur. J. Inorg. Chem. 2009 (2009) 5527-5534.

[3] M. M. Dîrtu, C. Neuhausen, A. D. Naik, A. Rotaru, L. Spinu, Y. Garcia, Insights into the origin of cooperative effects in the spin transition of $\left[\mathrm{Fe}\left(\mathrm{NH}_{2} \mathrm{trz}\right)_{3}\right]\left(\mathrm{NO}_{3}\right)_{2}$ : the role of supramolecular interactions evidenced in the crystal structure of $\left[\mathrm{Cu}\left(\mathrm{NH}_{2} \operatorname{trz}\right)_{3}\right]\left(\mathrm{NO}_{3}\right)_{2} \cdot \mathrm{H}_{2} \mathrm{O}, \mathrm{Inorg}$. Chem. 49 (2010) 5723-5736.

[4] A. Bousseksou, G. Molnár, L. Salmon, W. Nicolazzi, Molecular spin crossover phenomenon: recent achievements and prospects, Chem. Soc. Rev. 40 (2011) 3313-3335.

[5] M. Cavallini, Status and perspectives in thin films and patterning of spin crossover compounds, Phys. Chem. Chem. Phys. 14 (2012) 1186711876.

[6] P. Gütlich, H. Goodwin (Eds.), Spin Crossover in Transition Metal Compounds I-III, no. 233-235 in Top. Curr. Chem., Springer-Verlag, Berlin/Heidelberg, 2004.

[7] M. A. Halcrow (Ed.), Spin-Crossover Materials: Properties and Applications, Wiley, Chichester, 2013.

[8] P. Gütlich, A. Hauser, H. Spiering, Thermal and optical switching of iron(ii) complexes, Angew. Chem. Int. Ed. Engl. 33 (1994) $2024-2054$.

[9] A. Desaix, O. Roubeau, J. Jeftic, J. G. Haasnoot, K. Boukheddaden, E. Codjovi, J. Linarès, M. Noguès, F. Varret, Light-induced bistability in spin transition solids leading to thermal and optical hysteresis, Eur. Phys. J. B 6 (1998) 183-193.

[10] P. Gütlich, V. Ksenofontov, A. B. Gaspar, Pressure effect studies on spin crossover systems, Coord Chem Rev 249 (2005) 1811-1829.

[11] J. A. Real, A. B. Gaspar, M. C. Muñoz, Thermal, pressure and light switchable spin-crossover materials, Dalton Trans 2005 (2005) 20622079.

[12] Iu. Gudyma, A. Maksymov, M. Dimian, Stochastic kinetics of photoinduced phase transitions in spin-crossover solids, Phys. Rev. E 88 (2013) 042111.

[13] V. Martínez, Z. A. Castillo, M. C. Muñoz, A. B. Gaspar, J.-F. Etrillard, C Létard, S. A. Terekhov, G. V. Bukin, G. Levchenko, J. A. Real, Thermal-, pressure- and light-induced spin-crossover behaviour in the two-dimensional hofmann-like coordination polymer [Fe(3clpy $)_{2} \mathrm{Pd}(\mathrm{CN})_{4}$ ], Eur. J. Inorg. Chem. 2013 (2013) 813-818.

[14] O. Kahn, E. Codjovi, Y. Garcia, P. J. van Koningsbruggen, R. Lapouyade, L. Sommier, Spin-transition molecular materials for display and data processing, in: M. M. Turnbull, T. Sugimoto, L. K. Thompson (Eds.), Molecule-Based Magnetic Materials: Theory, Techniques, and Applications, American Chemical Society, Washington, 1996, pp. 298-310.

[15] G. Molnár, S. Cobo, J. A. Real, F. Carcenac, E. Daran, C. Vieu, A. Bousseksou, A combined top-down/bottom-up approach for the nanoscale patterning of spin-crossover coordination polymers, Adv. Mater. 19 (2007) 2163-2167.

[16] P. Gamez, J. S. Costa, M. Quesada, G. Arom, Iron spin-crossover compounds: from fundamental studies to practical applicattions, Dalton Trans. 2009 (2009) 7845-7853.

[17] P. Gütlich, A. B. Gaspar, Y. Garcia, Spin state switching in iron coordination compounds, Beilstein J. Org. Chem. 9 (2013) $342-391$.

[18] G. Molnár, L. Salmon, W. Nicolazzi, F. Terki, A. Bousseksou, Emerging properties and applications of spin crossover nanomaterials, J. Mater. Chem. C 2 (2014) 1360-1366.

[19] R. Jeon, J. G. Park, C. R. Haney, T. D. Harris, Spin crossover iron(II) complexes as PARACEST MRI thermometers, Chem. Sci. 5 (2014) 2461-2465.

[20] J. Wajnflasz, Etude de la transition „Low Spin” - „High Spin” dans les complexes octaédriques dion de transition, Phys. Stat. Sol. 40 (1970) 537-545.

[21] A. Bousseksou, J. Nasser, J. Linares, K. Boukheddaden, F. Varret, Ising-like model for the two-step spin-crossover, J. Phys. I France 2 (1992) 1381-1403.

[22] M. Buron-Le Cointe, J. Hébert, C. Baldé, N. Moisan, L. Toupet, P. Guionneau, J. F. Létard, E. Freysz, H. Cailleau, E. Collet, Intermolecular control of thermoswitching and photoswitching phenomena in two spin-crossover polymorphs, Phys. Rev. B 85 (2012) 064114.

[23] M. Nishino, S. Miyashita, Effect of the short-range interaction on critical phenomena in elastic interaction systems, Phys. Rev. B 88 (2013) 014108.

[24] Iu. Gudyma, A. Maksymov, C. Enachescu, Phase transition in spin-crossover compounds in the breathing crystal field model, Phys Rev B 89 (2014) 224412.

[25] Iu. Gudyma, V. Ivashko, J. Linares, Diffusionless phase transition with two order parameters in spin-crossover solids, J. Appl. Phys. 116 (2014) 173509.

[26] H. Cailleau, M. Lorenc, M. Guérin, M. Serrvol, E. Collet, M. Buron-Le Cointe, Structural dynamics of photoinduced molecular switching in the solid state, Acta Cryst. A 66 (2010) 189-197.

[27] S. Nozawa, T. Sato, M. Chollet, K. Ichiyanagi, A. Tomita, H. Fujii, S. Adachi, S. Koshihara, Direct probing of spin state dynamics coupled with electronic and structural modifications by picosecond time-resolved xafs, J. Am. Chem. Soc. 132 (2010) 61-63.

[28] Yu. Gudyma, O. Semenko, Nonequilibrium kinetics in spin-crossover compounds, Phys. Status Solidi B 241 (2) (2004) 370-376.

[29] Iu. Gudyma, A. Maksymov, High spin metastable state relaxation of spin-crossover solids driven by white noise, J. Phys. Chem. Solids 72 (2011) 73-77. 